\title{
Adaptation to Flooding in the Low-Income Urban Settlements of the Least Developed Countries
}

\author{
Anika N. Haque \\ Department of Geography, University of Cambridge \\ Cambridge, United Kingdom \\ anikanasra@gmail.com
}

\section{Extended Abstract}

Changes in the climate system are evident and are projected to continue over the next century. This will worsen the existing environmental problems of numerous countries, particularly the Least Developed Countries as they lack the capacity to protect their cities, and, the impacts of climatic variability are expected to impose a disproportionately high risk on poorer groups. Urban adaptation to climate change has been relatively underexamined, with clear gaps in research into urban vulnerability. Mainstream, top-down adaptation approaches are being questioned, as they have failed to reduce vulnerability, especially of the most vulnerable members of society. The importance of addressing micro- level adaptive capacity of low-income urban settlers has become apparent, as they face the greatest challenges to adapt and are also the most exposed. The overall research objective is to understand adaptation processes of low-income urban populations to one climate-related risk (i.e. flooding), as well as to enhance and develop new knowledge about ways in which the adaptation to climate change amongst these populations can be better tackled at different levels - by households and communities and also to identify how the relevant agencies and organizations can effectively contribute to the adaptation process. Hence the research spans the range from bottom-up to top-down, and attempts to understand bottom-up behaviours in order to improve the way in which top-down interventions can be made more effective.

The case study, Dhaka, the capital of Bangladesh, is one of the fastest growing megacities in the world. It is highly susceptible to flooding because of its physical setting. The most risk associated with flooding is in the low-lying areas of the city in the east. This also acts as a water retention area for the whole city, but these retention areas are being encroached to accommodate the rapidly increasing population, which in turn hampers the natural drainage of the city. Moreover, there is a lack of drainage infrastructure and the area is unprotected from flooding. Dhaka East was almost completely inundated in recent floods, and was inundated for a longer period of time than other parts of the city.

Both primary and secondary data have been used to conduct the research. The selection of the specific data collection methods was primarily governed by the different scales to be covered, i.e. household, community and organization, and the resulting data requirements. Hence questionnaire survey, focus group discussions and interviews were applied. Based on the analysis of the vulnerability and adaptation system, the research has developed a grounded theory on the processes whereby adaptive behaviour is learned and diffused in bottom-up ways amongst the population at risk, and how more top-down procedures to contribute to this can be improved (i.e. positively influenced). Therefore, a combined 'grounded theory-systems analysis' approach has been developed to analyze the diverse data. The research resulted with a strategic and operational framework integrating the bottom up and top down approaches that provide new knowledge and guidance as to how the low-income settlements can better adapt to the climatic changes. In doing so, it has identified the influencing factors of the adaptation process and how those factors influence the overall system. It also reveals how the adaptation process is influenced if there is interaction between the low-income group and relevant organizations. 